\title{
The feasibility of incentivizing participation in an online social network weight loss program
}

\author{
Sherry Pagoto, PhD \\ University of Massachusetts Medical School \\ Sherry.pagoto@umassmed.edu \\ Effie Olendzki, MBA \\ University of Massachusetts Medical School \\ Effie.olendzki@umassmed.edu \\ Christine May, PhD \\ University of Massachusetts Medical School \\ Christine.may@umassmed.edu
}

\begin{abstract}
Engagement in online social network-delivered weight loss interventions is a predictor of weight loss. Incentivizing engagement in a subset of participants may increase group engagement and subsequent weight loss. In a pilot feasibility trial, 56 adults with obesity were randomized to two Facebook-delivered weight loss interventions, one had 10\% users incentivized to engage daily and the other did not. We compared conditions on engagement and weight loss, and then compared incentivized users and natural high engagers on weight loss. Participants were 46.3 (SD: 10.3) years and 89\% female. The incentivized user condition had greater total engagement ( $p=0.0361)$, but weight loss did not differ ( $p=0.2096)$. Three natural superusers emerged in each condition. Natural superusers lost more weight than incentivized users ( $p=0.0358)$. Natural superusers' posts elicited more comments than incentivized superusers ( $p=0.0107)$. Incentivized superusers may engage differently than natural superusers. Future studies should explore ways to promote engagement in online interventions.
\end{abstract}

\section{Introduction}

Social media use is reaching ubiquity with $74 \%$ of internet users in the US having at least one social network account.[1,2] While online social networks have traditionally been used as a way to connect with friends and family, users are now using them as a source of health information and to connect with others who have the same health condition.[3] In

\author{
Molly Waring, PhD \\ University of Massachusetts Medical School \\ Molly.waring@umassmed.edu \\ Jessica Oleski, MA \\ University of Massachusetts Medical School \\ Jessica.oleski@umassmed.edu \\ Martinus Evans, MA \\ University of Massachusetts Medical School \\ martinus.evans@umassmed.edu
}

fact, large patient communities have organically developed online which may provide a critical mass for health promotion interventions.[4,5] For example, online social networks are increasingly being researched as a modality for delivering behavioral weight loss interventions.[6] Some studies have used online social networks as an adjunct to clinic-based weight loss programs, either by conducting scheduled group chats online[7,8] or by providing a message board/forum for participants to submit questions and chat.[9,10] Other studies have used online social networks as the main delivery modality for weight loss interventions. [5,11,12] Two systematic reviews of 12 and 20 studies, respectively, revealed that such interventions have shown modest outcomes.[6,13] Only one study isolated the impact of the online social network, showing that an online exercise intervention that included an online social network outperformed the same intervention without the online social network.[14]

Because participants have no visits to attend in an online social network delivered intervention, program engagement is defined as any visible act of participation including views, "likes," comments, posts, or shares. Engagement is important because intervention receipt requires posts to be viewed, read, and comprehended.[15] The literature has not established the level of engagement in an online social network intervention adequate to produce knowledge acquisition, facilitate social support, or ultimately, results in meaningful weight loss.[16] Online social network delivered weight loss studies show wide ranges of engagement, from some participants not engaging at all to others engaging multiple times per day.[5,15,17,18] While studies 
consistently show that engagement in the online social network predicts greater weight loss,[5,19-21] it is unclear whether this relationship is because weight loss success makes people more likely to engage, because engagement is a driver of weight loss, or because of a third variable that drives both weight loss and engagement. Regardless, increasing participant engagement may facilitate greater social support for weight loss and increase opportunities for feedback, which could conceivably improve outcomes. However, how to enhance engagement in online social network program remains an open question.

Monetary incentives have been used with success to enhance attendance in traditionally delivered weight loss interventions.[22,23] In an online social network delivered intervention, incentives could be used to enhance engagement in the network, specifically liking, posting, and replying to coach or fellow participant's posts. Incentivizing engagement might facilitate participant's emotional and intellectual investment in the intervention content and increase opportunities for social support and feedback. For example, if participants are incentivized to reply to posts, they may be more likely to read the posts as it would be necessary to compose a reply. Replying to fellow participants might also strengthen ties between participants. The drawback of incentives is that they may not be feasible in real world settings that are often resourcelimited. Inexpensive ways to leverage the power of incentives are needed.

Incentivizing every participant may not be necessary to increase engagement in an online social network since increased engagement by a few network members may stimulate engagement in the others via role modeling and social support processes. One study of two large online social network-based tobacco programs with over 32,000 registrants found that a small number of users $(0.4 \%$ $1.1 \%)$ whom they refer to as "superusers" accounted for $34.8 \%-46.2 \%$ of all content.[24] The investigators note that superusers, by playing a leadership and peer support role, have a powerful influence on the sustainability of the online social network. Another study of an online social network for smoking cessation also identified superusers, referring to them as "network integrators," who are instrumental to engaging other participants.[25] Such individuals emerge naturally in large online social networks, but rates are so low (about 1\%) that they may not routinely emerge in smaller $(n=20-40)$ online social networks designed to deliver behavioral counseling. For this reason, incentivizing a subset of users to play the role of superusers might bolster participation in these smaller groups.

In the present pilot study, using a randomized design, we tested the feasibility of incentivizing a small number of participants to become "superusers" in an online social network weight loss program. In one condition, $10 \%(n=3)$ of participants were randomly chosen to receive incentives to post regularly in a 12-week online social network weight loss program, while in the other condition no participants were incentivized to post regularly. The primary outcome was engagement among nonincentivized users in both treatment conditions. We also report retention and weight loss, although not powered to detect group differences, weight loss.

Although incentivizing engagement may increase engagement by some users, it is not clear if extrinsically-motivated posts are as engaging as those that are intrinsically-motivated, i.e., "natural superusers" who are highly engaged without being incentivized to do so. Thus, we secondarily examined the emergence of natural superusers, and compared level of engagement, the engagement their posts elicited (i.e., comments and likes from other users), and weight loss among incentivized and natural superusers. This allowed us to evaluate how similar incentivized superusers were to natural superusers.

\section{Methodology}

\subsection{Recruitment and Screening Procedures}

Participants were recruited from the local community around Worcester, MA. Recruitment procedures included electronic ads, community flyers, mass e-mails, and local newspaper ads. Electronic ads were posted on craigslist.org, our institution's internal website and e-newsletter, and electronic community newsletters. Mass e-mails were sent to participants signed up for the UMass Conquering Disease database, which is a program that informs patients about research studies that are actively recruiting. Community flyers were posted in local gyms, supermarkets, and vitamin shops.

Interested adults called or emailed our office to inquire about the study. A telephone screening was performed to provide the participant with details about the study and to assess initial eligibility criteria. Inclusion criteria included smartphone users currently using Facebook, interested in losing weight, and with a body mass index (BMI) of $25-45 \mathrm{~kg} / \mathrm{m}^{2}$. Participants were excluded if they were not interested, had diabetes, did not complete a baseline visit, had an inflexible schedule, were unable to travel to campus to complete the study visits, had 
participated in a previous weight loss study in our laboratory, were taking medication known to cause weight gain, had bariatric surgery, had a medical condition that limits physical activity or diet, did not have a primary care provider, was currently breastfeeding or pregnant, did not have a scale at home, had plans on starting another diet program, or did not speak English.

We received 252 inquiries, of which 84 adults were eligible to complete a baseline appointment that included informed consent, physical measurements of height and weight, physician approval, and completion of self-report measures. Of the 73 adults who completed a baseline assessment, 56 were eligible to be randomized into the study.

Participants were randomly assigned to one of two conditions, Superuser (SU) and Regular User (RU). Both conditions received the same 12-week weight loss intervention delivered in a secret Facebook group. The only difference between conditions was that the SU condition included 3 "superusers" who received financial compensation to engage on a daily basis to role model active engagement and provide social support to other participants. Except for receiving financial incentives, superusers did not differ from other participants. The first 4 eligible participants were asked to be superusers. One declined and was then not included in the study given this individual was no longer blind to the study purpose. All other participants were randomly assigned to either the SU or RU condition using an SPSS-generated randomization sequence.

The superusers were instructed not to tell other participants that they were receiving compensation for posting regularly. While we incentivized three superusers, none of the three were told of the other incentivized users in the group. No other participants in either the SU or RU group were informed that there were superusers in the SU group. Deception was used to insure that participants perceived the superuser posts as natural and genuine.

\subsection{Intervention}

The 12-week weight loss intervention provided was based on the Diabetes Prevention Program (DPP) Lifestyle Intervention.[26] Participants received a personalized calorie goal that would facilitate a 1-2 lb weight loss weekly and were encouraged to engage in 175 minutes of moderate physical activity per week. All participants were encouraged to use MyFitnessPal ${ }^{\circledR}$ to track diet and physical activity. Group material was translated into Facebook posts or online articles that were linked to in a Facebook post. Each day at $7 \mathrm{am}$ and $4 \mathrm{pm}$, posts relevant to the topic of the week were made by the coach. The same intervention content was posted in each condition using the social media management software, Buffer $($ C. Two weight loss coaches were assigned to each condition and were instructed to log in twice per day to reply to participant posts, answer participant questions, ask participants how they were doing, and otherwise facilitate conversations. One coach in each condition had a doctoral degree in psychology, while the other two had backgrounds in health education and personal training, respectively. While coaches knew of the existence of incentivized superusers, they were blind to whether their group included incentivized superusers.

\subsubsection{Superuser Instructions}

In the SU condition, superusers were instructed to make at least 2 original posts per week, reply to the coaches' posts and/or comment on other participants' posts at least once per day. This would result in a total of 9 posts per week or 1.28 posts or comments per day. This rate insured that each superuser modeled a daily presence and that all program posts (2/day) received replies without causing the program posts to get buried in user posts. In terms of what to post, superusers were instructed to post successes or difficulties they are having losing weight, questions for the group or coaches, updates about their progress, recipes they tried, photos of meals they cooked, and/or links to local events/resources. They were informed that in our previous studies, these were the types of posts that participants enjoyed seeing from other participants. Superusers received $\$ 25$ for completing this assignment each week. Full compensation for the week was provided for $2+$ posts and 7+ comments per week. If superusers were falling behind on their posting assignment for the week on Friday, research staff prompted the individual by email. On average, superusers posted at least 9 posts/comments on 11.3 (SD: 0.6 ) out of 12 weeks.

\subsection{Follow-Up Visit}

Follow-up assessments were scheduled during week 13 and included completion of an online survey and a brief in-person visit to measure weight. All participants received $\$ 50$ compensation at the followup. The superusers received \$300 (\$25 per week) at the follow-up visit.

\subsection{Debriefing}


Participants were debriefed about the incentivized superusers in focus groups after the follow-up visit. Non-incentivized participants in both conditions were informed that 3 participants were randomly selected to receive financial incentives to post regularly as a way to increase engagement. Participants in the RU condition were informed that these 3 participants were in the other condition. One participant expressed dismay at the use of incentives for engagement because of the possibility that it produced non-genuine conversations. No other participants expressed concern.

\subsection{Measures}

\subsubsection{Engagement}

Engagement data was extracted from the Facebook Applications Programming Interface (API) at the end of the intervention period with a program developed specifically for this purpose. One participant in the SU condition deleted her Facebook account after the intervention so we were unable to retrieve her engagement data. Engagement data was available for 55 participants (98\%). Engagement was defined as original posts by a participant, comments on other's (coach or participant) posts, and "liking” other's posts or comments.

\subsubsection{Retention}

Retention was defined as completion of the followup assessment.

\subsubsection{Weight loss}

Weight was measured in the lab by research staff using a calibrated balance beam scale at baseline and follow-up. Two participants who missed the followup visit provided weight via self-report. Percent weight loss was calculated by dividing pounds lost at follow-up by baseline weight and multiplying by 100 . We also calculated the percent of participants in each condition who lost clinically significant weight (i.e., $\geq 5 \%$ ).

\subsubsection{Natural Superusers}

Natural superusers were defined as nonincentivized participants in either condition who made an average of 9 or more posts or comments per week, equivalent to the frequency of engagement required of incentivized superusers.

\section{Analytic Plan}

As engagement variables were not normally distributed, we used Wilcoxon rank-sum tests to compare engagement across conditions and among subgroups of participants. Chi-square tests were used to compare the treatment conditions on categorical baseline characteristics and retention rates. Mean age and baseline BMI were compared across condition using t-tests. For participants missing weight at follow-up $(n=2)$, we assumed no weight change (baseline value carried forward). Comparisons of percent weight loss used independent sample t-tests for continuous weight loss and chi-square tests for weight loss of $5 \%$ or greater. Spearman correlations were used to examine the association between engagement and weight loss.

\section{Results}

Participant characteristics in relation to treatment condition are shown in Table 1. No characteristics differed significantly by treatment condition.

Table 1: Baseline characteristics by treatment condition, M(SD) or \%

\begin{tabular}{|l|c|c|}
\hline & $\begin{array}{c}\text { RU condition } \\
(\mathrm{n}=26)\end{array}$ & $\begin{array}{c}\text { SU condition } \\
(\mathrm{n}=30)\end{array}$ \\
\hline BMI $\left(\mathrm{kg} / \mathrm{m}^{2}\right)$ & $31.6(4.2)$ & $33.4(5.3)$ \\
\hline Age (years) & $45.5(10.8)$ & $47.0(9.9)$ \\
\hline Female & 88 & 90 \\
\hline Non-Hispanic white & 92 & 90 \\
\hline Married & 58 & 73 \\
\hline$\geq$ Bachelor's degree & 46 & 67 \\
\hline
\end{tabular}

\subsection{Engagement}

Among participants who were not incentivized to engage, significant differences were observed by condition in total engagement $(p=0.0361)$ and likes $(p=0.0480)$, but not number of posts $(p=0.2483)$ or comments $(\mathrm{p}=0.1133$; Table 2$)$.

Table 2: Engagement among nonincentivized users by treatment condition, M(SD)

\begin{tabular}{|l|c|c|c|}
\hline & $\begin{array}{c}\text { RU } \\
(\mathrm{n}=26)\end{array}$ & $\begin{array}{c}\text { SU } \\
(\mathrm{n}=26)\end{array}$ & $\begin{array}{c}\mathrm{p}- \\
\text { value }\end{array}$ \\
\hline $\begin{array}{l}\text { Total } \\
\text { engagements }\end{array}$ & $117(151)$ & $194(205)$ & $\mathbf{0 . 0 3 6 1}$ \\
\hline Posts & $8(19)$ & $6(6)$ & 0.2483 \\
\hline Comments & $41(52)$ & $49(35)$ & 0.1133 \\
\hline
\end{tabular}




\begin{tabular}{|l|l|l|l|}
\hline Likes & $68(90)$ & $140(180)$ & $\mathbf{0 . 0 4 8 0}$ \\
\hline
\end{tabular}

\subsection{Retention}

Two participants were lost to follow-up in the RU condition (92\% retention), and none were lost to follow-up in the SU condition (100\% retention), a difference that was not significantly different between conditions $(\mathrm{p}=0.12)$.

\subsection{Weight Loss}

No significant difference was observed in percent weight loss by treatment condition $(-1.9 \%$ in RU condition versus $-3.2 \%$ in SU condition, $\mathrm{p}=0.1539$; Table 3). Percent weight loss also did not differ by treatment condition when excluding incentivized superusers $(-3.4 \%$ in SU condition, $\mathrm{p}=0.1096$; Table 3 ). One incentivized superuser gained $0.5 \%$ from baseline while the other two lost $0.2 \%$ and $4.0 \%$, for a mean of $1.2 \%$ lost (SD: $2.4 \%)$. No differences were observed by condition in the percent of participants losing $5 \%$ or greater weight when incentivized users were included ( $\mathrm{p}=0.7570$; Table 3 ), or when incentivized users were not included $(p=0.5886$; Table 3).

Table 3. Retention and Weight Loss by Condition, M(SD) or \%

\begin{tabular}{|l|c|c|c|}
\hline & $\begin{array}{c}\text { RU } \\
\text { condition }\end{array}$ & \multicolumn{2}{|c|}{ SU condition } \\
\hline & $\begin{array}{c}\text { All } \\
(\mathrm{n}=26)\end{array}$ & $\begin{array}{c}\text { All } \\
(\mathrm{n}=30)\end{array}$ & $\begin{array}{c}\text { Non- } \\
\text { incentivized } \\
\text { only (n=27) }\end{array}$ \\
\hline Retention & 92 & 100 & 100 \\
\hline \% weight loss & $-1.9(3.7)$ & $-3.2(3.1)$ & $-3.4(3.1)$ \\
\hline $\begin{array}{l}\text { Percent losing } \\
\geq 5 \%\end{array}$ & 23 & 27 & 30 \\
\hline
\end{tabular}

Among all non-incentivized participants in both conditions, total engagement was significantly correlated with percent weight loss $(\mathrm{r}=-0.50$, $\mathrm{p}=0.0002)$. Number of comments $(\mathrm{r}=-0.41, \mathrm{p}=0.0024)$ and number of likes $(r=-0.47, p=0.0004)$ were correlated with weight loss, but number of original posts was not $(\mathrm{r}=-0.17, \mathrm{p}=0.2282)$.

\subsection{Natural Superusers}

Three natural superusers emerged in each treatment condition. Engagement by natural superusers did not differ by condition: total engagement ( $\mathrm{p}=1.000)$, posts $(\mathrm{p}=0.1904)$, comments $(\mathrm{p}=0.3827)$, and likes $(\mathrm{p}=0.3827)$. Natural superusers' engagement (i.e., posts, comments, likes, total) did not differ from incentivized superusers (Table 4). However, natural superusers lost significantly greater weight on average than incentivized superusers (-5.3\% versus- $1.2 \%$, $\mathrm{p}=0.0358$; Table 4$)$.

Table 4: Engagement and weight loss among natural and incentivized superusers, $M(S D)$ or $\%$

\begin{tabular}{|l|c|c|c|}
\hline & $\begin{array}{c}\text { Natural } \\
\text { superusers } \\
(\mathrm{n}=6)\end{array}$ & $\begin{array}{c}\text { Incentivized } \\
\text { superusers } \\
(\mathrm{n}=3)\end{array}$ & $\mathrm{p}$-value \\
\hline $\begin{array}{l}\text { Total } \\
\text { engagement }\end{array}$ & $502(174)$ & $680(651)$ & 0.6985 \\
\hline Posts & $27(34)$ & $33(10)$ & 0.3662 \\
\hline Comments & $135(45)$ & $205(100)$ & 0.1198 \\
\hline Likes & $340(187)$ & $442(549)$ & 0.5186 \\
\hline $\begin{array}{l}\% \text { weight } \\
\text { loss }\end{array}$ & $-5.3(2.2)$ & $-1.2(2.4)$ & $\mathbf{0 . 0 3 5 8}$ \\
\hline$\geq 5 \%$ loss & 50 & 0 & 0.1336 \\
\hline
\end{tabular}

On average, posts by natural superusers and incentivized superusers attracted similar rates of total engagement $(\mathrm{p}=0.2396)$, comments $(\mathrm{p}=0.1655)$, and likes $(p=0.9774)$ from participants. However, posts by natural superusers attracted more comments ( $p=0.0107)$ from non-incentivized participants than did posts by incentivized superusers. Neither number of likes $(p=0.9774)$ nor total engagement $(p=0.2324)$ by non-incentivized participants differed.

\section{Discussion}

Incentivizing $10 \%$ of participants to engage regularly in an online social network-delivered weight loss intervention resulted in greater total engagement among other participants compared to a condition in which no users were incentivized. This difference appeared to be driven by "likes," given the two conditions did not differ on original posts or comments. Participants in the SU condition "liked" on average more than twice the number of posts and comments as participants in the RU condition. Incentivizing engagement among a few participants appears to elicit positive reinforcement of posts, but perhaps did not start discussions, as would have been evidenced by greater comments and posts in that condition. Overall, this study showed that incentivizing superusers was feasible and acceptable, but might not elicit more meaningful conversation among other participants, which is what was hypothesized could possibly drive greater weight loss outcomes. 
In terms of weight loss outcomes, the condition with incentivized superusers did not lose significantly more weight in spite of greater engagement, although the study was not powered to detect differences in weight loss. Mean weight loss ranged from $-3.4 \%$ to $2.0 \%$ over 12 weeks of intervention. To put this weight loss in context, the gold standard DPP lifestyle intervention produced 6.9\% weight loss over 24 weeks.[27] Weekly weight loss in this study ($0.28 \%$ per week in the SU condition) was comparable for the DPP (-0.28\% per week), suggesting that an online social network delivered program may have the potential to be as efficacious as face-to-face counseling interventions. Fully powered trials are needed to explore this question given the potential that delivering intervention via commercial online social networks is lower in patient burden and cost.

In both conditions, three natural superusers emerged; these were individuals who posted as frequently as the incentivized superusers. Incentivized superusers lost significantly less weight than other participants and less weight than natural superusers. One possibility for less weight loss in incentivized superusers is that the role of incentivized posting was distracting for people who may not be naturally inclined to take on such a role. Given only 3 participants were incentivized to engage, further research is needed to firmly establish the impact of incentivized engagement on weight loss. In a recent smoking cessation study using Facebook, no differences were found in smoking cessation for participants who were incentivized to comment on all posts versus those who were not incentivized to comment.[28] Another possibility for lower weight loss among incentivized superusers is that incentivized posts were driven by obligation and thus may not have been of the nature that created opportunities for support or feedback. Future research should explore the characteristics of participant posts that inspire greater engagement among other participants, and those associated with greater weight loss by the participant himself or herself.

Greater weight loss among natural superusers relative to incentivized superusers may have been due to their posts being more likely to solicit feedback and support. Engagement in the online social network might also be a proxy of engagement in the behavioral weight loss program such that people who engaged on the Facebook page may have also had read more of the articles and posts, and had better adherence to the behavioral recommendations. Alternatively, their weight loss success may have made posting updates less intimidating which led them to post more often. Those who struggled most with weight loss might have felt too embarrassed to post about it. Future research should explore the timing and patterns and engagement in relation to weight loss progress during an online social network delivered weight loss intervention.

Online weight loss programs are becoming increasingly popular due to their convenience and emerging data showing effectiveness.[29-31] Participants of such programs may need guidance on how best to use a weight loss social network. Several studies now show that greater engagement predicts greater weight loss, $[5,10,18]$ but it is not clear what type of engagement is meaningful. Incentivizing engagement does not necessarily facilitate "meaningful" engagement as evidenced by our data showing 2 out of 3 of incentivized superusers did not lose weight. Concerns about the possibility that financial incentives reduce intrinsic motivation have been raised in the literature.[32] We used incentives in one specific way; incentives could conceivably be more impactful if used in other ways. For example, awards could be given for participating in specific behavioral challenges (e.g., 10K steps per day, 7 days of self-monitoring) or for achieving outcomes (e.g., $5 \%$ weight loss). Research on what types of engagement in the online social network is most meaningful in terms of promoting weight loss are needed. Also needed are strategies to increase such meaningful engagement.

\subsection{Limitations}

The present study has several limitations. First, it is a pilot study so was not powered to detect differences in weight loss by condition. Our goal was to explore whether having some incentivized superusers would increase overall engagement in their condition. One related limitation is that 3 incentivized superusers may not have been enough or may have been too much to produce the type of effect on engagement that would then result in weight loss in that condition. In observational studies of large online social networks, about $0.4-7.6 \%$ of participants were classified as highly engaged users.[24,33] Because our network size was smaller than these studies, we chose a $10 \%$ rate of incentivized superusers to make sure we had enough superusers in case some did not adhere to the posting schedule. Another limitation is that the treatment period was half the length of standard weight loss programs thus it is possible that engagement may have increased further past 12 weeks and perhaps weight loss differences would then emerge. Finally, we did not predict how many natural superusers would emerge and their presence may have further limited our ability to see differences in engagement 
and weight loss across conditions. Our study showed that about $11 \%$ of participants of an online social network intervention may be natural superusers, slightly higher than the $0.4-7.6 \%$ observed in previous research,[24,33] though the criteria for high engagement differs across studies. A stricter definition of superuser would likely reduce the number of natural superusers. In future studies, the definition of natural superuser might also include criteria related to type of content included in posts or comments, as certain content might be more influential and elicit more conversation than others.

\subsection{Conclusion}

The present study found that incentivizing $10 \%$ of participants in an online social network-delivered weight loss intervention resulted in more engagement than a comparison group in which no users were incentivized to engage. However, the incentivized participants lost less weight than natural superusers who emerged during the program suggesting incentivizing engagement in an online social network may produce a qualitatively different type of engagement than that of participants who are naturally high engagers. Future studies should explore strategies to increase meaningful engagement among low engagers in an online social networkdelivered health promotion program to fully realize the potential of these networks for behavior change.

\section{References}

[1] Social Media Update 2013. PEW Internet and American Life Project, 2013. at

http://www.pewinternet.org/2013/12/30/social-mediaupdate-2013/. Archived at http://www.webcitation.org/6b64FaV8V )

[2] Social Networking Fact Sheet. 2014. at http://www.pewinternet.org/fact-sheets/social-networkingfact-sheet/. Archived at

http://www.webcitation.org/6ZVvgfc4q.)

[3] Peer-to-peer Healthcare 2011. 2012, at

http://www.pewinternet.org/2011/02/28/peer-to-peerhealth-care-2/. Archived at

http://www.webcitation.org/6b65r4Dy0.)

[4] Attai DJ, Cowher MS, Al-Hamadani M, Schoger JM, Staley AC, Landercasper J. Twitter Social Media is an Effective Tool for Breast Cancer Patient Education and Support: Patient-Reported Outcomes by Survey. J Med Internet Res 2015;17:e188.

[5] Pagoto SL, Waring ME, Schneider KL, et al. TwitterDelivered Behavioral Weight-Loss Interventions: A Pilot Series. JMIR research protocols 2015;4:e123.

[6] Ashrafian H, Toma T, Harling L, Kerr K, Athanasiou T, Darzi A. Social networking strategies that aim to reduce obesity have achieved significant although modest results. Health Aff (Millwood) 2014;33:1641-7.

[7] Womble LG, Wadden TA, McGuckin BG, Sargent SL, Rothman RA, Krauthamer-Ewing ES. A randomized controlled trial of a commercial internet weight loss program. Obes Res 2004;12:1011-8.

[8] Harvey-Berino J, West D, Krukowski R, et al. Internet delivered behavioral obesity treatment. Prev Med 2010;51:123-8.

[9] Morgan PJ, Lubans DR, Collins CE, Warren JM, Callister R. The SHED-IT randomized controlled trial: evaluation of an Internet-based weight-loss program for men. Obesity (Silver Spring, Md 2009;17:2025-32. [10] Hales SB, Davidson C, Turner-McGrievy GM. Varying social media post types differentially impacts engagement in a behavioral weight loss intervention. Transl Behav Med 2014;4:355-62.

[11] Jane M, Foster J, Hagger M, Pal S. Using new technologies to promote weight management: a randomised controlled trial study protocol. BMC Public Health 2015;15:509.

[12] Patrick K, Marshall SJ, Davila EP, et al. Design and implementation of a randomized controlled social and mobile weight loss trial for young adults (project SMART). Contemp Clin Trials 2014;37:10-8.

[13] Chang T, Chopra V, Zhang C, Woolford SJ. The role of social media in online weight management: systematic review. J Med Internet Res 2013;15:e262.

[14] Zhang J, Brackbill D, Yang S, Centola D. Efficacy and causal mechanism of an online social media intervention to increase physical activity: Results of a randomized controlled trial. Prev Med Rep 2015;2:651-7.

[15] Merchant G, Weibel N, Patrick K, et al. Click "like" to change your behavior: a mixed methods study of college students' exposure to and engagement with Facebook content designed for weight loss. J Med Internet Res 2014;16:e158.

[16] Pagoto S, Waring ME, May CN, et al. Adapting Behavioral Interventions for Social Media Delivery. J Med Internet Res 2016;18:e24.

[17] Napolitano MA, Hayes S, Bennett GG, Ives AK, Foster GD. Using Facebook and text messaging to deliver a weight loss program to college students. Obesity (Silver Spring, Md 2013;21:25-31.

[18] Turner-McGrievy GM, Tate DF. Weight loss social support in 140 characters or less: use of an online social network in a remotely delivered weight loss intervention. Transl Behav Med 2013;3:287-94.

[19] Gold BC, Burke S, Pintauro S, Buzzell P, HarveyBerino J. Weight loss on the web: A pilot study comparing a structured behavioral intervention to a commercial program. Obesity (Silver Spring, Md 2007;15:155-64. [20] Webber KH, Tate DF, Michael Bowling J. A randomized comparison of two motivationally enhanced Internet behavioral weight loss programs. Behav Res Ther 2008;46:1090-5.

[21] Turner-McGrievy GM, Beets MW, Moore JB, Kaczynski AT, Barr-Anderson DJ, Tate DF. Comparison of traditional versus mobile app self-monitoring of physical activity and dietary intake among overweight adults 
participating in an mHealth weight loss program. J Am Med Inform Assoc 2013;20:513-8.

[22] Rothberg AE, McEwen LN, Kraftson AT, et al. Factors associated with participant retention in a clinical, intensive, behavioral weight management program. BMC obesity 2015;2:11.

[23] Sykes-Muskett BJ, Prestwich A, Lawton RJ, Armitage CJ. The utility of monetary contingency contracts for weight loss: a systematic review and meta-analysis. Health psychology review 2015;9:434-51.

[24] van Mierlo T, Voci S, Lee S, Fournier R, Selby P. Superusers in social networks for smoking cessation: analysis of demographic characteristics and posting behavior from the Canadian Cancer Society's smokers' helpline online and StopSmokingCenter.net. J Med Internet Res 2012;14:e66.

[25] Cobb NK, Graham AL, Abrams DB. Social network structure of a large online community for smoking cessation. Am J Public Health 2010;100:1282-9.

[26] Diabetes Prevention Program Research G. The Diabetes Prevention Program (DPP): description of lifestyle intervention. Diabetes Care 2002;25:2165-71.

[27] Wing RR, Hamman RF, Bray GA, et al. Achieving weight and activity goals among diabetes prevention program lifestyle participants. Obes Res 2004;12:1426-34. [28] Ramo DE, Thrul J, Chavez K, Delucchi KL, Prochaska JJ. Feasibility and Quit Rates of the Tobacco Status Project: A Facebook Smoking Cessation Intervention for Young Adults. J Med Internet Res 2015;17:e291.

[29] Sepah SC, Jiang L, Peters AL. Translating the Diabetes Prevention Program into an Online Social Network: Validation against CDC Standards. Diabetes Educ 2014;40:435-43.

[30] Turner-McGrievy GM, Campbell MK. Nutrition information to the desktop: a pilot online nutrition course on saturated fat for public librarians increases knowledge, expectancies, and self-efficacy. J Nutr Educ Behav 2009;41:188-93.

[31] Willis EA, Szabo-Reed AN, Ptomey LT, et al. Do weight management interventions delivered by online social networks effectively improve body weight, body composition, and chronic disease risk factors? A systematic review. J Telemed Telecare 2016.

[32] Hagger MS, Keatley DA, Chan DC, et al. The goose is (half) cooked: a consideration of the mechanisms and interpersonal context is needed to elucidate the effects of personal financial incentives on health behaviour. Int $\mathrm{J}$ Behav Med 2014;21:197-201.

[33] Healey B, Hoek J, Edwards R. Posting behaviour patterns in an online smoking cessation social network: implications for intervention design and development. PLoS One 2014;9:e106603. 